EPJ Web of Conferences 53, 06004 (2013)

DOI: $10.1051 /$ epjconf/20135306004

(C) Owned by the authors, published by EDP Sciences, 2013

\title{
Deflection of ultra-high energy heavy nuclei in the Galactic magnetic field
}

\author{
Gwenael Giacinti ${ }^{1,2, a}$, Michael Kachelrieß², Dmitri Semikoz ${ }^{3}$ and Günter Sigl $\left.\right|^{4}$ \\ ${ }^{1}$ Clarendon Laboratory, University of Oxford, Parks Road, Oxford OX1 3PU, UK \\ ${ }^{2}$ Institutt for fysikk, NTNU, Trondheim, Norway \\ ${ }^{3}$ AstroParticle and Cosmology (APC), Paris, France \\ ${ }^{4}$ II. Institut für Theoretische Physik, Universität Hamburg, Hamburg, Germany
}

\begin{abstract}
In this work, we investigate the impacts a potential shift towards a heavy composition at the end of the cosmic ray (CR) spectrum would have on the future data [1,2]. We present detailed simulations for the propagation of ultra-high energy (UHE) heavy nuclei, with $E \geq 60 \mathrm{EeV}$, within recent Galactic magnetic field (GMF) models. We consider both regular and turbulent components of the GMF. We show that with UHE heavy nuclei, there is no one-to-one correspondence between the arrival directions of cosmic rays measured at Earth and the direction of their extragalactic sources. Sources can have several distorted images on the sky. We compute images of galaxy clusters and of the supergalactic plane in recent GMF models and show the challenges, but also the possibilities, of "ultra-high energy cosmic ray astronomy" with heavy nuclei. Finally, we present a quantitative study of the impact of the GMF on the (de-)magnification of source fluxes, due to magnetic lensing. Such effects cannot be neglected in case of heavy primaries.
\end{abstract}

\section{INTRODUCTION}

The composition of the ultra-high energy cosmic ray (UHECR) flux at the highest energies $E \gtrsim 10^{19} \mathrm{eV}$ is the subject of current debate. Hadronic physics must be extrapolated from lower energies and complex data analyses for different experiments are compatible with either light primaries - HiRes [3] and Telescope Array [4] - or a shift towards heavier ones - Pierre Auger Observatory [5, 6] and Yakutsk EAS array muon data [7].

In this work, we study the propagation of UHE heavy nuclei in recent Galactic magnetic field (GMF) models, and discuss some of the consequences a shift towards heavier nuclei would have. For clarity, we consider here only iron nuclei with energies $E \geq 60 \mathrm{EeV}$. The extrapolation of the following results to a mixed composition is straightforward. Most studies have focused on proton or light nuclei primaries at the highest energies, with the exception of Refs. [1, 2, 8-14].

Qualitatively, several important effects would arise for heavy nuclei primaries. Contrary to UHE proton sources, one can expect UHE heavy nuclei sources to have several distorted images on the celestial sphere, even at the highest energies. In general, there is no one-to-one correspondence between the UHE heavy nuclei arrival directions as measured at Earth and the directions of their extragalactic sources. Also, one can expect fluxes from such sources to be significantly magnified or demagnified due to magnetic lensing effects in the GMF. At $60 \mathrm{EeV}$, iron nuclei sources located in up to one fifth of the sky would not be detectable by the next generation of experiments due to the strong demagnification of their fluxes. In the future, the difference of propagation in the GMF between light and heavy primaries

\footnotetext{
ae-mail: gwenael.giacinti@physics.ox.ac.uk
}

This is an Open Access article distributed under the terms of the Creative Commons Attribution License 2.0, which permits unrestricted use, distribution, and reproduction in any medium, provided the original work is properly cited. 


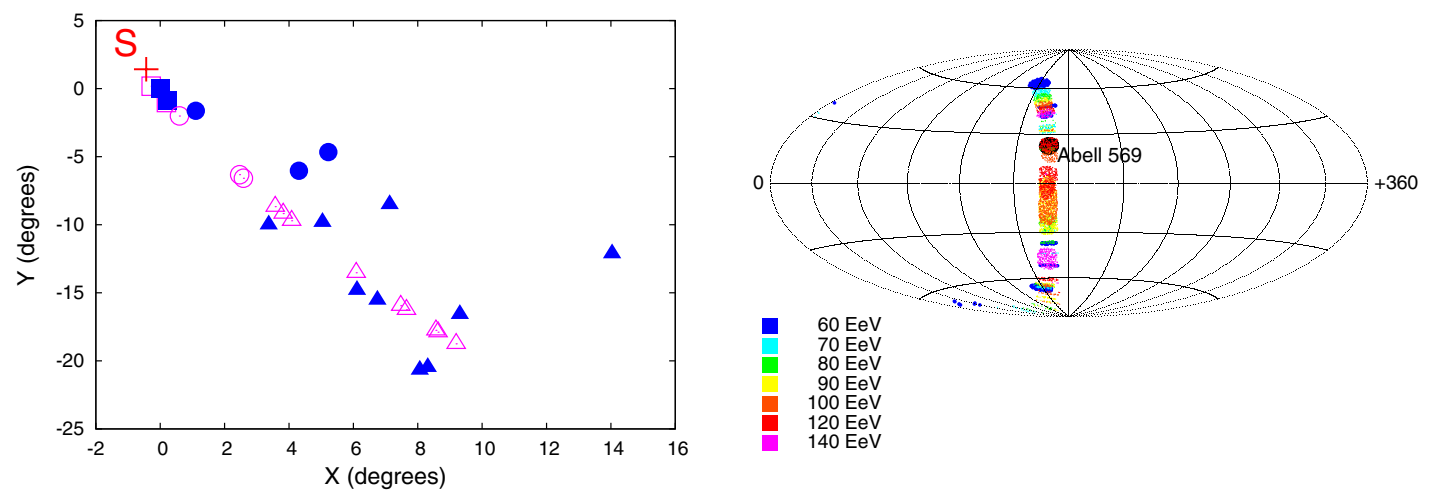

Figure 1. Left panel: portion of the celestial sphere, with a proton source " $\mathrm{S}$ " (red cross) and the arrival directions of its simulated events with energies $E$. Triangles for $10 \mathrm{EeV} \leq E \leq 20 \mathrm{EeV}$, circles for $20 \mathrm{EeV} \leq E \leq 40 \mathrm{EeV}$ and squares for $E \geq 40 \mathrm{EeV}$. Magenta open symbols for deflections in the GMF regular component only, and blue filled symbols when the turbulent field is also taken into account; Right panel: image, in Galactic coordinates, of the galaxy cluster Abell 569 emitting $E=60-140 \mathrm{EeV}$ iron nuclei, see key for colours. ASS version of the PTKN model for the regular GMF, and $B_{0}=0$. Black disk for the position of the cluster on the sky.

will be important to understand sky maps of UHECR arrival directions. It may give further information on the CR composition at the highest energies.

In Section 2, we present the main generic features of UHE heavy nuclei source images and investigate the challenges of source detection. In Section 3, we discuss quantitatively the impact of magnetic lensing in the GMF.

\section{IMAGES OF UHECR SOURCES}

The Galactic magnetic field can be modelled as the sum of a regular (large scale fluctuations) and a turbulent (smaller scale fluctuations) component. We use the "PS" [15, 16], "Sun08" [17], "Sun08MH" [1], "PTKN" [18] and "Jansson-Farrar 2012" [19] models for the regular GMF. We model the turbulent component, as in Refs. [2, 20, 21] and take the profile introduced in Ref. [22] for its root mean square (rms) strength $B_{\mathrm{rms}}$. Let us denote $B_{0}$ its rms strength at Earth and $z_{0}$ its lateral extension in the Galactic halo. The directions on the sky in which CRs are deflected, as well as the (de-)magnification of a given source flux -see Section 3- strongly depend on the GMF model. However, averaged quantities such as the average UHECR deflection angle and fractions of the sky with a given (de-)magnification, are much less model dependent. In this sense, the following results are generic.

Extragalactic magnetic fields are expected to be stronger in the large scale structure of the Universe (LSS), and significantly weaker in voids. UHECR deflections in such fields are poorly constrained [23]. Deflections in models of extragalactic magnetic fields range from negligible [24, 25] to more than ten degrees, even for $100 \mathrm{EeV}$ protons [26, 27]. In the first scenario, UHE heavy nuclei would still be significantly deflected inside the galaxy clusters that contain their sources, but would only experience negligible deflections between the clusters and our Galaxy. Therefore, clusters containing UHE heavy nuclei sources would shine as a whole and can be considered as extended sources in this scenario. In the following, we address this case by computing images of different nearby galaxy clusters and consider them as $\simeq 5^{\circ}$ extended sources. In the second scenario, UHE heavy nuclei would be strongly deflected in the whole local large scale structure, making it shine as a whole. In a first approximation, we can study this case by computing the supergalactic plane image in the GMF.

Figure 1 (left panel) shows the positions on the sky of a proton source "S" (red cross) and of its events with $E \geq 10^{19} \mathrm{eV}$-triangles for events with energies $E \leq 20 \mathrm{EeV}$, circles for $20 \mathrm{EeV} \leq E \leq 40 \mathrm{EeV}$ 

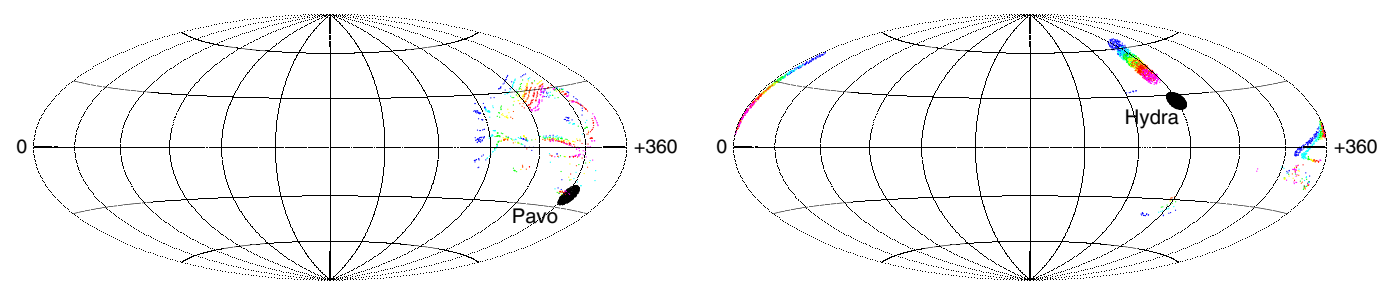

Figure 2. Images, in Galactic coordinates, of Pavo (left panel) and Hydra (right panel) galaxy clusters emitting $E=60-140 \mathrm{EeV}$ iron nuclei. PS model for the regular GMF and $B_{0}=0$. Black disks for the positions of the clusters on the sky. Same colour code as in Fig. 1 (right panel).

and squares for $E \geq 40 \mathrm{EeV}$. When considering only a regular field, CRs are deflected along a curve (magenta open symbols) and their angular distance to the source is approximately proportional to $1 / E$. Adding a turbulent component slightly spreads the events (blue filled symbols). Events at the highest energies, $E \geq 40 \mathrm{EeV}$, are not deflected more than a few degrees from the source. Such an example is representative of proton and light nuclei source images [22, 28], except for sources located in the Galactic plane and towards the Galactic center.

On the contrary, for heavy nuclei primaries, there is in general no one-to-one correspondence between directions on the sky of CRs before they enter the Galaxy and their arrival directions at Earth. As a consequence, UHE heavy nuclei sources usually have multiple images, even at the highest energies. We present in Figure 1 (right panel) the image of the galaxy cluster Abell 569 in the ASS version of the PTKN model for the regular GMF, emitting iron with energies ranging from $60 \mathrm{EeV}$ to $140 \mathrm{EeV}$. Adding the turbulent GMF would spread more the image. In this model, the cluster displays at least seven images at $60 \mathrm{EeV}$ (blue patches on the sky). Also, they are more or less distorted, and distances of individual events to the source do not satisfy the $1 / E$ ordering: For instance, the $140 \mathrm{EeV}$ image on the lower part of Figure 1 (right panel) is further from the source than one of the $60 \mathrm{EeV}$ images. While the image of a given cluster strongly depends on the GMF model, such global characteristics are generic for UHE heavy nuclei sources. In general, a better knowledge of the GMF than currently available is necessary to detect such sources and reconstruct their positions on the sky. However, GMF models are expected to be improved in the future thanks to LOFAR and SKA [29, 30]. In a few unfavorable cases, when source images are strongly spread over the celestial sphere, an improved GMF model may still not be sufficient. The image of Pavo galaxy cluster in the PS model is one of such examples, see Fig. 2 (left panel). Nonetheless, a few favourable cases in which one could detect some sources without an improved GMF model may exist. Ref. [31] showed that in some GMF models, and for some source positions on the sky, at least one image looks like a proton source image enlarged by a factor $\approx Z$ for nuclei of charge $Z$. For instance, the energy ordering of events in the main image of Hydra cluster in the PS model roughly follows that of proton sources, see Fig. 2 (right panel).

If UHECRs suffer significant deflections in the large scale structure, their directions before entering our Galaxy would not correlate with their sources, but would still correlate with the LSS. We plot in Fig. 3 the $60 \mathrm{EeV}$ iron images, in the PS model, of the supergalactic plane. As a first approximation, we model the supergalactic plane as a $\pm 10^{\circ}$ stripe in supergalactic latitude. In the left panel, the turbulent field is set to zero and in the right panel, it is assumed to have a $B_{0}=4 \mu \mathrm{G}$ strength at Earth and a $z_{0}=3 \mathrm{kpc}$ extension in the Galactic halo. One can see that in both cases, the distribution of CR arrival directions at Earth is anisotropic. Therefore, a heavy composition at the highest energies is in principle not incompatible with detecting an anisotropy in the UHECR arrival directions. For a large turbulent field strength $B_{0} \approx 10 \mu \mathrm{G}$ and a large extension in the halo $z_{0} \approx 10 \mathrm{kpc}$, arrival directions at Earth of $60 \mathrm{EeV}$ iron nuclei from the supergalactic plane are distributed significantly more isotropically on the sky than in Fig. 3 (right panel). 

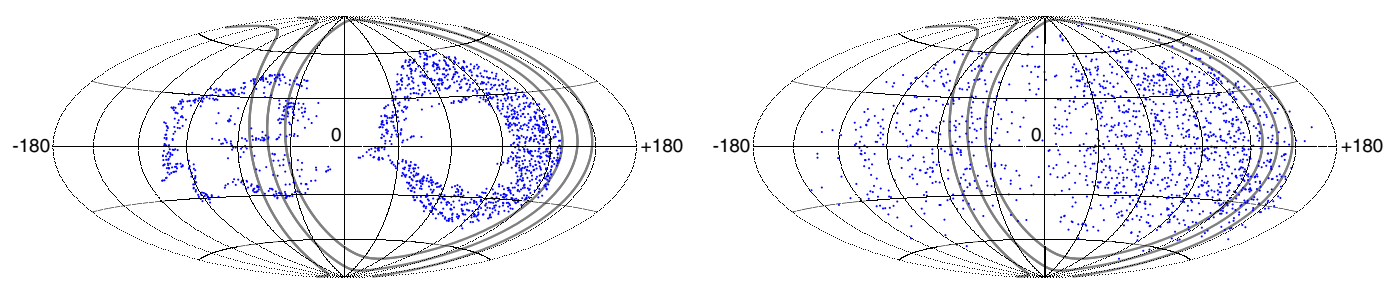

Figure 3. Images on the sky of the supergalactic plane emitting $60 \mathrm{EeV}$ iron nuclei (blue dots). PS model for the regular GMF. Left panel: with no additional turbulent component; Right panel: with an additional turbulent field of strength $B_{0}=4 \mu \mathrm{G}$ and extension in the halo $z_{0}=3 \mathrm{kpc}$. Maps are in Galactic coordinates. Grey lines correspond to supergalactic latitudes $-10^{\circ}, 0^{\circ}$ and $+10^{\circ}$.
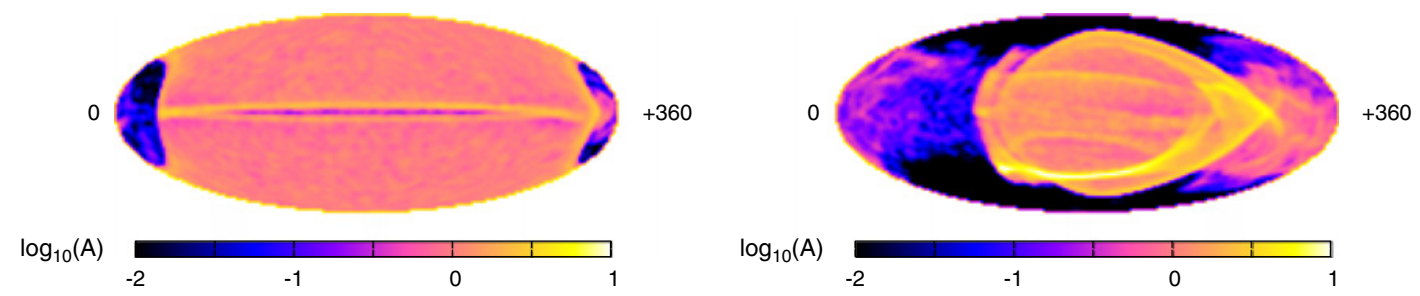

Figure 4. Logarithm of the amplification factor $A$ of fluxes from $60 \mathrm{EeV}$ proton (left panel) and $60 \mathrm{EeV}$ iron nuclei (right panel) extragalactic sources, depending on their positions on the sky. PS model for the regular GMF and no additional turbulent field $\left(B_{0}=0\right)$. Maps are in Galactic coordinates.

\section{EFFECTS OF MAGNETIC LENSING IN THE GALACTIC MAGNETIC FIELD}

The Galactic magnetic field (de-)magnifies the fluxes from individual UHECR sources, due to magnetic lensing effects. Let us denote $A$ the "amplification factor" of a given point source, and $F$ the flux received at Earth from that source. If the GMF were set to zero, one would receive at Earth a flux $F / A$ from the same source. If $A>1$ (respectively $A<1$ ), the source flux is said to be magnified by a factor $A$ (respectively demagnified by a factor $1 / A$ ).

Figure 4 presents maps, in Galactic coordinates, of the amplification factor $A$ in the PS model for $60 \mathrm{EeV}$ proton (left panel) and iron nuclei (right panel) extragalactic sources, versus their positions on the sky. The GMF turbulent component is set to zero, and plots have been computed as in Ref. [1]. The two panels are significantly different : For protons, $A \simeq 1$ in most of the sky (orangish-magenta colour), while for iron nuclei, $1 / 3 \leq A \leq 3$ in only about one half of the sky. In general, magnetic lensing effects are negligible for light nuclei primaries, except in a few directions towards the Galactic plane and center, see dark region towards $l \approx 0^{\circ}$ in the left panel. On the contrary, such effects cannot be neglected in most of the sky for UHE heavy nuclei sources, even for those far from the Galactic plane. In Fig. 4 (right panel), sources located in the small white region of the sky would have their fluxes amplified by a factor $A \gtrsim 10$, while those located in the large black regions would have their fluxes demagnified by more than 100 . The positions on the sky of these regions are model dependent. Sources located in directions where $A \leq 1 / 100$ cannot be detected neither by present nor by future UHECR observatories. We find that for $60 \mathrm{EeV}$ iron nuclei primaries, such regions encompass up to about one fifth of the sky in recent regular GMF models. Let us also note that the value of $A$ in one given direction of the sky depends on the rigidity $E / Z$. Therefore, the spectrum detected at Earth for one given source may be distorted by the GMF. 

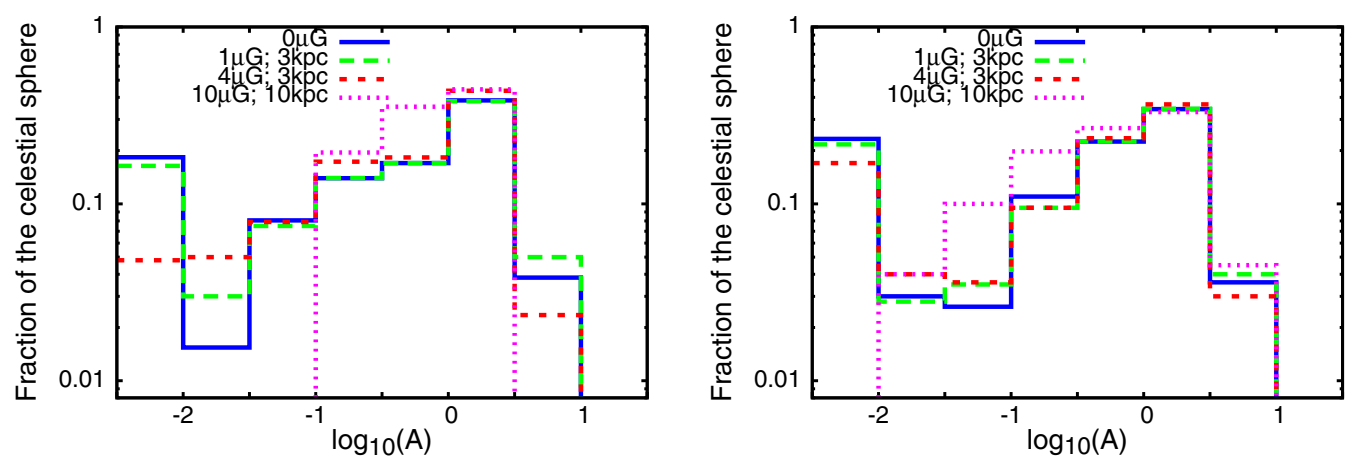

Figure 5. Histograms of fractions of the extragalactic sky with given amplification factors, for $60 \mathrm{EeV}$ iron nuclei. Bins of width $\Delta \log _{10}(A)=0.5$. PS (left panel) and Sun08 (right panel) models for the GMF regular component. Blue lines for no additional turbulent field $\left(B_{0}=0\right)$, and green, red and magenta lines respectively for $\left\{B_{0} ; z_{0}\right\}=\{1 \mu \mathrm{G} ; 3 \mathrm{kpc}\},\{4 \mu \mathrm{G} ; 3 \mathrm{kpc}\}$ and $\{10 \mu \mathrm{G} ; 10 \mathrm{kpc}\}$. On both panels, the first bin corresponds to $\log _{10}(A) \leq-2$.

Adding a turbulent component to the regular GMF smooths the sharp features in Figure 4 and globally reduces the extension on the sky of regions with large magnification or demagnification. The fraction of the celestial sphere in which $A \sim 1$ grows for stronger and more extended turbulent fields. Since the turbulent field strength and extension in the halo are not well constrained, we test several combinations of $B_{0}$ and $z_{0}$. Histograms of fractions of the extragalactic sky with given amplification factors $A$ are shown in Figure 5 (left panel) for the PS model. The blue solid line corresponds to results for no additional turbulent field. The green, red, and magenta lines respectively correspond to $\left\{B_{0}=\right.$ $\left.1 \mu \mathrm{G} ; z_{0}=3 \mathrm{kpc}\right\},\left\{B_{0}=4 \mu \mathrm{G} ; z_{0}=3 \mathrm{kpc}\right\}$, and a more extreme case $\left\{B_{0}=10 \mu \mathrm{G} ; z_{0}=10 \mathrm{kpc}\right\}$. This illustrates how regions with large (de-)magnification shrink with increasing turbulent field contributions. The fraction of the sky with $1 / 3 \leq A \leq 3$ increases to $\simeq 80 \%$ for the case $\left\{B_{0}=10 \mu \mathrm{G} ; z_{0}=10 \mathrm{kpc}\right\}$. While the value of $A$ in one given direction on the sky strongly depends on the GMF model, the distribution of fractions of the sky with given factors $A$ is less model dependent. Figure 5 (right panel) shows results for the Sun08 GMF model. They are globally comparable with those for the PS model. In both cases, regions with $A \leq 1 / 100$ disappear for the strongest turbulent field we tested (magenta lines). Source searches would however not be facilitated by strong turbulence, because their images would be significantly spread over the celestial sphere.

\section{CONCLUSIONS AND PERSPECTIVES}

We have propagated UHE iron nuclei with energies $E \geq 60 \mathrm{EeV}$ in recent Galactic magnetic field models, including both regular and turbulent components. We have pointed out that several important effects cannot be neglected in case of heavy primaries at the highest energies.

We have shown in Section 2 that heavy nuclei sources would generally display multiple and distorted images on the celestial sphere. The energy ordering of events in such images can be far from that expected for proton sources. For some sources, lower energy events can be closer to the source than higher energy events. In most cases, a better knowledge of the GMF than currently available would be needed to detect sources and reconstruct their positions accurately. In the future, LOFAR and SKA data will put better constraints on the Galactic magnetic field geometry and properties [29, 30]. In some of the GMF models tested here, heavy nuclei sources located in some directions on the sky display at least one "enlarged proton-like" image. With sufficient statistics, detecting such sources -if any exists- is possible with the present knowledge of the GMF. 
We have addressed in Section 3 (de-)magnification of fluxes from individual sources, due to magnetic lensing in the GMF. While such effects can be ignored for proton primaries -except in a few directions towards the Galactic center and plane-, they are substantial for heavy nuclei primaries. One important finding is that UHE heavy nuclei sources would have their fluxes demagnified by a factor $\geq 100$ in up to about one fifth of the extragalactic sky. Such sources cannot be detected, even by the next generation of UHECR experiments. The fraction of the sky covered by these regions shrinks for stronger and more extended GMF turbulent components, while the spread on the sky of individual source images increases.

GG acknowledges funding from the European Research Council under the European Community's Seventh Framework Programme (FP7/2007-2013) / ERC grant agreement no. 247039, as well as support from NTNU Trondheim (Norway) and from the Research Council of Norway through an Yggdrasil grant. GS acknowledges support from the Deutsche Forschungsgemeinschaft through the collaborative research centre SFB 676, and from the State of Hamburg through the Collaborative Research program "Connecting Particles with the Cosmos", from the "Helmholtz Alliance for Astroparticle Phyics HAP", funded by the Initiative and Networking Fund of the Helmholtz Association.

\section{References}

[1] G. Giacinti, M. Kachelrieß, D. V. Semikoz and G. Sigl, JCAP 1008 (2010) 036

[2] G. Giacinti, M. Kachelrieß, D. V. Semikoz and G. Sigl, Astropart. Phys. 35 (2011) 192

[3] P. Sokolsky, PoS ICHEP 2010 (2010) 444

[4] Y. Tsunesada [for the Telescope Array Collaboration], "Highlights from Telescope Array," arXiv:1111.2507 [astro-ph.HE]

[5] J. Abraham et al. [Pierre Auger Observatory Collaboration], Phys. Rev. Lett. 104 (2010) 091101

[6] P. Facal et al. [for the Pierre Auger Collaboration], "The Pierre Auger Observatory II: Studies of Cosmic Ray Composition and Hadronic Interaction models," arXiv:1107.4804 [astro-ph.HE]

[7] A. V. Glushkov, I. T. Makarov, M. I. Pravdin, I. E. Sleptsov, D. S. Gorbunov, G. I. Rubtsov and S. V. Troitsky, JETP Lett. 87 (2008) 190

[8] D. Harari, S. Mollerach and E. Roulet, JHEP 9908 (1999) 022

[9] D. Harari, S. Mollerach and E. Roulet, JHEP 0010 (2000) 047

[10] D. Harari, S. Mollerach and E. Roulet, JHEP 0002 (2000) 035

[11] H. Takami and K. Sato, Astrophys. J. 724 (2010) 1456

[12] S. Vorobiov et al., Nucl. Phys. Proc. Suppl. 196 (2009) 203

[13] G. Golup, D. Harari, S. Mollerach and E. Roulet, JCAP 1107 (2011) 006

[14] R. -Y. Liu, X. -Y. Wang, W. Wang and A. M. Taylor, Astrophys. J. 755 (2012) 139

[15] M. Prouza and R. Smida, Astron. Astrophys. 410 (2003) 1

[16] M. Kachelrieß, P. D. Serpico and M. Teshima, Astropart. Phys. 26 (2006) 378

[17] X. H. Sun, W. Reich, A. Waelkens and T. Enslin, Astron. Astrophys. 477 (2008) 573

[18] M. S. Pshirkov, P. G. Tinyakov, P. P. Kronberg and K. J. Newton-McGee, Astrophys. J. 738 (2011) 192

[19] R. Jansson and G. R. Farrar Astrophys. J. 757 (2012) 14

[20] D. De Marco, P. Blasi and T. Stanev, JCAP 0706 (2007) 027

[21] G. Giacinti, M. Kachelriess, D. V. Semikoz and G. Sigl, JCAP 1207 (2012) 031

[22] G. Giacinti, X. Derkx and D. V. Semikoz, JCAP 1003 (2010) 022

[23] K. Kotera and A. V. Olinto, Ann. Rev. Astron. Astrophys. 49 (2011) 119

[24] K. Dolag, D. Grasso, V. Springel and I. Tkachev, JETP Lett. 79 (2004) 583 [Pisma Zh. Eksp. Teor. Fiz. 79 (2004) 719]

[25] K. Dolag, D. Grasso, V. Springel and I. Tkachev, JCAP 0501 (2005) 009

[26] G. Sigl, F. Miniati and T. A. Ensslin, Phys. Rev. D 70 (2004) 043007 


\section{UHECR 2012}

[27] G. Sigl, F. Miniati and T. Ensslin, Nucl. Phys. Proc. Suppl. 136 (2004) 224

[28] G. Golup, D. Harari, S. Mollerach and E. Roulet, Astropart. Phys. 32 (2009) 269

[29] B. M. Gaensler, R. Beck and L. Feretti, New Astron. Rev. 48 (2004) 1003

[30] R. Beck and B. M. Gaensler, New Astron. Rev. 48 (2004) 1289

[31] G. Giacinti and D. V. Semikoz, Phys. Rev. D 83 (2011) 083002 